two breaks in any particular two of the four strands present at the zygotene stage (zygophase) coinciding, then the chance for such coincidence in any two of all four strands is $6 / x$. One-third of these cases of coincidence will be between sister strands of the same chromosome. Hence the chance for coincidence between two breaks at a particular point in the twin halves of opposed homologous chromosomes is $4 / x$. (The chance for coincidence between four such breaks, one in each of the four strands at pachyphase, is only $1 / x^{2}$ at any particular point.)

In any coincidence of two breaks in strands of different homologues, for which the chance at any particular point is $4 / x$, we may suppose that in half the cases the ends of the same threads are reunited, and in the other half of the cases ends of different threads unite, thus forming a chiasma. Hence the chance of a chiasma, or point of segmental interchange, between homologues at any one point in the bivalent is $2 / x$. Since there is no chiasma between the other two strands, $1 / x$ represents the chance of segmental interchange at any one point in the chromosomes resulting from the maturation division. (The occurrence of a second, or even a third, chiasma is to be allowed for.)

Hence $\mathrm{I} / x=c / 100$, where $c$ is the observed percentage number of cross-overs between two adjacent genes.

John Belling.

Dept. of Genetics,

Carnegie Institution of Washington, Cold Spring Harbor,

Long Island, N.Y., Jan. 21.

\section{Optically Excited Iodine Bands with Alternate Missing Lines.}

$W_{\mathrm{E}}$ have recently been studying, under improved conditions, the fluorescent bands which develop around the 'fundamental' doublets when iodine is excited, in the presence of helium, by the green mercury line. The use of a battery of four mercury are lamps surrounding the iodine tube excites such a brilliant fiuorescence that it is possible to photograph it in 24 hours in the second order of a 9-foot grating. On the plates so obtained it is apparent that only alternate lines of the corresponding absorption bands occur, namely, those for which $m^{\prime}$ is even. Now the fluorescent bands are known to be developed by collisions of the second kind between excited iodine molecules and helium atoms, wherein $m^{\prime}$ is changed from 34, the value originally excited, to various neighbouring values. The new data, then, show that the rotational quantum number of the excited iodine molecules can change only by even numbers during these collisions of the second kind.

This result, while incomprehensible on the classical Bohr-Lenz theory, is entirely in accord with the conelusions of the wave mechanics. According to the theory of Hund, successive rotational states of a symmetrical molecule, such as $I_{2}$, have, alternately, eigen-functions symmetric and antisymmetric in the two nuclei. Moreover, since the symmetric and antisymmetric eigen-functions correspond to different orientations of the spins of the two nuclei, and since these spins are presumably very loosely coupled, it is to be expected that transitions between symmetric and antisymmetric states will be very infrequent. In fact, Dennison has recently solved the long-outstanding problem of the specific heat of hydrogen by assuming that transitions between symmetric and antisymmetric states do not occur in appreciable numbers, even during the time it takes to make a measurement of specific heats.
The absence of the alternate lines in the optically excited iodine bands affords direct evidence in support of the theories of Hund and Dennison.

$\begin{array}{ll}\text { Johns Hopkins University, } & \text { R. W. Wood. } \\ \text { New York University. } & \text { F. W. Loomis. }\end{array}$

New York University.

\section{Absorption of X-Rays in Various Elements.}

Prof. Richtmyer, in a letter published in Nature of Dec. 24, 1927, p. 915, states that he has formerly tried the formula

$$
\delta_{K}=\frac{E_{K}}{E_{L_{1}}}
$$

for the ratio $\delta_{K}$ of the absorption cofficients at the $K$-discontinuity. In the paper cited by Prof. Richtmyer (Phys. Rev., 23, p. 292, Feb. 1924) he says: "This ratio is of the same order of magnitude as, but somewhat smaller than, the ratio of the energies required to remove a single $K$ and a single $L$ electron from an atom of the absorber." But the formula which contains the empirical facts, and given in my letter to NATURE of Nov. 12,1927, p. 695 , is not this one but

$$
\delta_{K}=\frac{E_{K}}{E_{L_{I}}}
$$

Numerically, this formula (2) differs considerably from (1).

As the notations may easily cause confusion, I wish to point out the different meanings of the two equations.

There exists three energy-levels in the $L$-group which have been designed by $L_{1}, L_{2}$, and $L_{3}$, where $L_{1}$ is much more marked than the two others. In the system of notation introduced by Bohr these levels were designated by $L_{\mathrm{III}}, L_{\mathrm{II}}$, and $L_{\mathrm{I}}$ respectively; that is, $L_{1}$ corresponds to $L_{\mathrm{III}}$ and $L_{3}$ to $L_{\mathrm{I}}$. The fact that $L_{1}$ (or $L_{I I I}$ ) is the most prominent level in the $L$-group might explain why the true formula (2) containing the less prominent level $L_{3}$ or $L_{\mathrm{I}}$ has up to this time escaped observation.

As will be seen from my doctorate dissertation, the experimental results found by Prof. Richtmyer also fit very well with the two Jaws described in my former letter.

EDvin Jönsson.

Physical Laboratory,

University, Upsala, Sweden, Jan. 9.

\section{Inflammable Gas from Plants.}

IN Prof. Findlay's letter in Nature of Jan. 14, he refers to Black's statement that "the Dictamnus Fraxinella emits " marsh gas "from its flowers," and asks for information on the topic.

Black undoubtedly alluded to Dictamnus albus, the fraxinella or dittany of southern Europe and central Asia, an old inhabitant of country gardens, which has showy, varicoloured, fragrant flowers. The plant is covered with glands that secrete a volatile oil, and in hot weather the air about the plant sometimes becomes inflammable therefrom.

According to Schimmel and Company (Geschäftsber, Oct. 1906) oil of white dittany contains 85 per cent. of pulegone, of which it smells strongly. Pulegone, or pulegenone, has been described by Semmler (Ber., 35, $1022 ; 1902$ ), and by Wallach (Ann., 329, $125 ; 1903$ ). W. A. HAMOR.

(Assistant Director.)

Mellon Institute of Industrial Research, Pittsburgh, $\mathrm{Pa}$. Jan. 30 .

No. 3043, VoL. 121] 